\title{
Oxacillin Sodium
}

National Cancer Institute

\section{Source}

National Cancer Institute. Oxacillin Sodium. NCI Thesaurus. Code C720.

The sodium salt form of oxacillin, a semisynthetic penicillinase-resistant and acid-stable penicillin with an antimicrobial activity. Oxacillin binds to penicillin-binding proteins in the bacterial cell wall, thereby blocking the synthesis of peptidoglycan, a critical component of the bacterial cell wall. This leads to inhibition of cell growth and causes cell lysis. 